\title{
Telomerase as a DNA-Dependent DNA Polymerase ${ }^{\dagger}$
}

\section{Supporting Information}

\author{
Jason D. Legassie and Michael B. Jarstfer* \\ University of North Carolina, School of Pharmacy, Division of Medicinal Chemistry
}

Chapel Hill, North Carolina 27599-7360

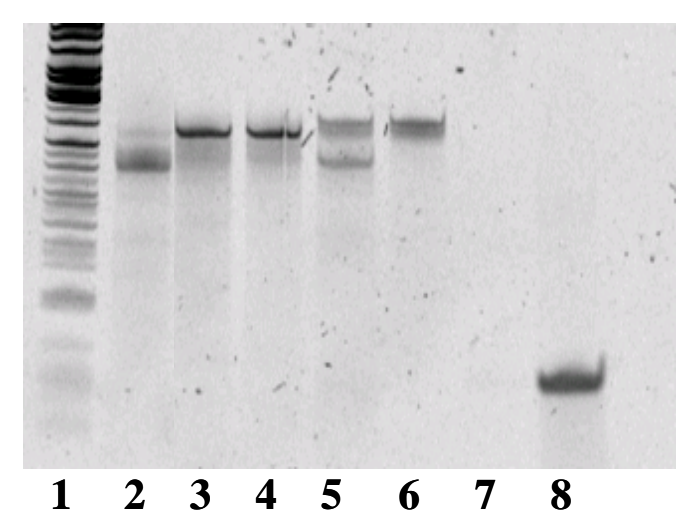

Figure S1: Digestion with RQ1 DNase demonstrates the presence of the DNA template in tTRd43-48. Nucleic acids (100 ng per reaction) were incubated at $37{ }^{\circ} \mathrm{C}$ for $30 \mathrm{~min}$ in 1X RQ1 DNase buffer in the presence of 1 Unit of RQ1 DNase (Promega) in a $10 \mu \mathrm{L}$ reaction. Control reactions were run in DNase buffer in the absence of DNase.

Reactions were stopped by the addition of $10 \mu \mathrm{L}$ of denaturing loading buffer. Samples were separated on a denaturing $10 \%$ polyacrylamide gel and stained with SYBR ${ }^{\circledR}$ Green II RNA gel stain (Molecular Probes) following the manufacturers protocol. The gel was imaged on a Molecular Dynamics Storm 860 phosphorimager. Lane 1, PhiX174

DNA/Hinf 1 (DNA Ladder; Promega); lane 2, tTR52-159; lane 3, tTR + DNase; lane 4, tTR; lane 5, tTRd43-48 + DNase; lane 6, tTRd43-48; lane 7, 50-nt control DNA Oligo + DNase; lane 8, 50-nt DNA Oligo 


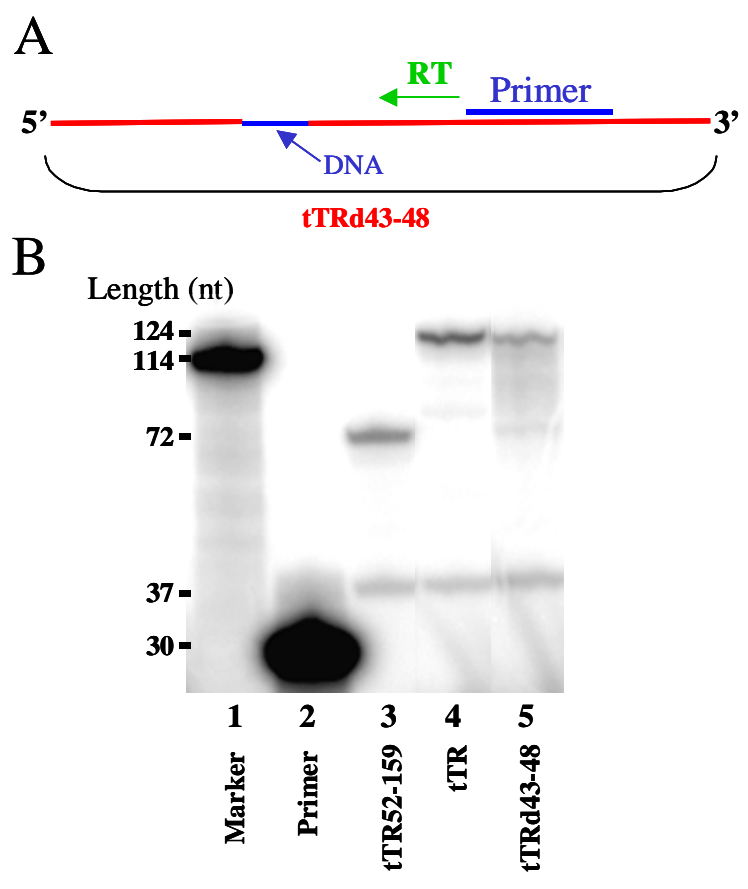

Figure S2: M-MLV reverse transcriptase is able to utilize tTRd43-48 as a template for DNA strand synthesis. (A) Schematic of tTRd43-48 annealed with a DNA primer (5'TGTCGATAGTCTTTTGTCCCGCATTACCAC) that is complementary to nucleotides 95-124 of tTR. The green arrow indicates the direction in which M-MLV RT extends the primer using tTR as the template. (B) Denaturing PAGE gel of product RT reactions with tTR52-159 (lane 3), tTR (lane 4), and tTRd43-48 (lane 5). Each reaction contained $60 \mathrm{ng}$ of target RNA, $500 \mu \mathrm{M}$ dNTP mix, $0.33 \mu \mathrm{M}\left[\alpha_{-}{ }^{32} \mathrm{P}\right]-\mathrm{dGTP}(3000 \mathrm{Ci} / \mathrm{mmol}), 1 \mu \mathrm{M}$ DNA primer, 20 Units RNasin (Promega), 1X M-MLV RT reaction buffer (Promega), and 100 Units of M-MLV RT (Promega) in $10 \mu \mathrm{L}$ total volume. All reactions were heated to $90{ }^{\circ} \mathrm{C}$ for $3 \mathrm{~min}$ and then cooled on ice for $10 \mathrm{~min}$, before the addition of RNasin and M-MLV RT. After addition of M-MLV RT, reactions were incubated at 37 ${ }^{\circ} \mathrm{C}$ for $30 \mathrm{~min}$. Reactions were stopped by the addition of $10 \mu \mathrm{L}$ of denaturing loading buffer. Products were separated by denaturing PAGE on a $10 \%$ polyacrylamide gel. The gel was dried and exposed overnight to a phosphorimager plate, which was imaged using a Molecular Dynamics Storm 860 phosphorimager. Lane 1, 5'-32 P-labeled 114-nt oligonucleotide size marker; lane $2,5^{\prime}-{ }^{32} \mathrm{P}$-labeled DNA primer; lane 3 , reverse transcription product from tTR52-159 as template (72-nt long); lane 4, reverse transcription product from tTR as template (124-nt long); lane 5, reverse transcription product from tTRd43-48 as template (124-nt long). 

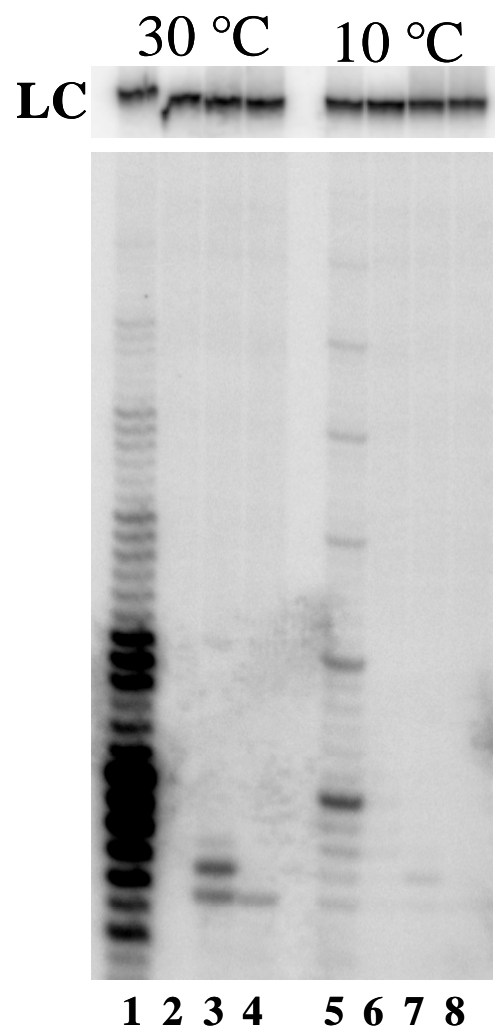

Figure S3: Temperature dependence of telomerase-catalyzed primer extension. Telomerase assays were carried out as described in Materials and Methods using primer p5 $\left(\mathrm{G}\left(\mathrm{T}_{2} \mathrm{G}_{4}\right)_{2} \mathrm{TTGG}\right)$, except that reactions were allowed to proceed for $90 \mathrm{~min}$.

Reactions in lanes 1-4 were incubated at $30^{\circ} \mathrm{C}$ and reactions in lanes 5-8 were incubated at $10^{\circ} \mathrm{C}$. Telomerases were assembled using wild type protein, tTERT, and either tTR, tTRd43-48, or tTRd43-51 as indicated. Lane 1, tTR; lane 2, tTR + RNase; lane 3, tTRd43-48; lane 4, tTRd43-51; lane 5, tTR; lane 6, tTR + RNase; lane 7, tTRd43-48; lane 8, tTRd43-51. + RNase indicates a sample pretreated with RNase A. A ${ }^{32}$ P-labeled 114nt oligonucleotide was used as a recovery and loading control (LC). 


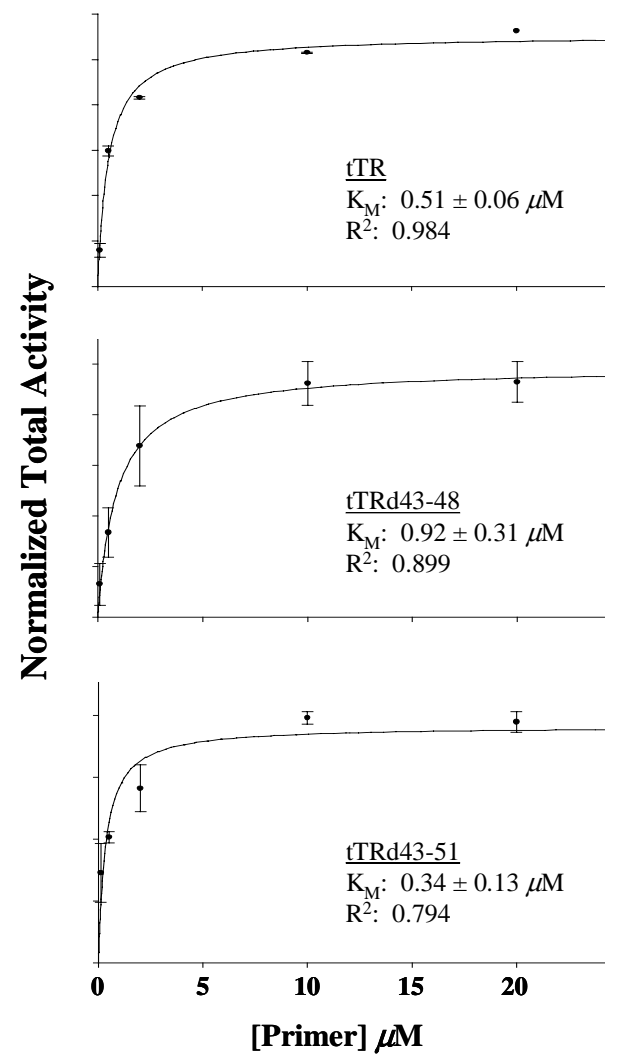

Figure S4: Michaelis-Menten plots for the determination of $\mathrm{K}_{\mathrm{M}}$ of primer $\mathrm{p} 5$ for tTR, tTRd43-48, and tTRd43-51 telomerases. Graphs were generated using SigmaPlot 2001 Version 7.0 with Enzyme Kinetics Module Version 1.1 using data derived from gel density measurements from two separate experiments. The total activity at each primer concentration was normalized to gel loading controls and to wild type activity levels. The gel in Figure 4 was one of two identical experiments used to determine $\mathrm{K}_{\mathrm{M}}$. Since accurate $\left[\alpha-{ }^{32} \mathrm{P}\right]$-dGTP specific activity was not determined, Vmax values were not obtained. 


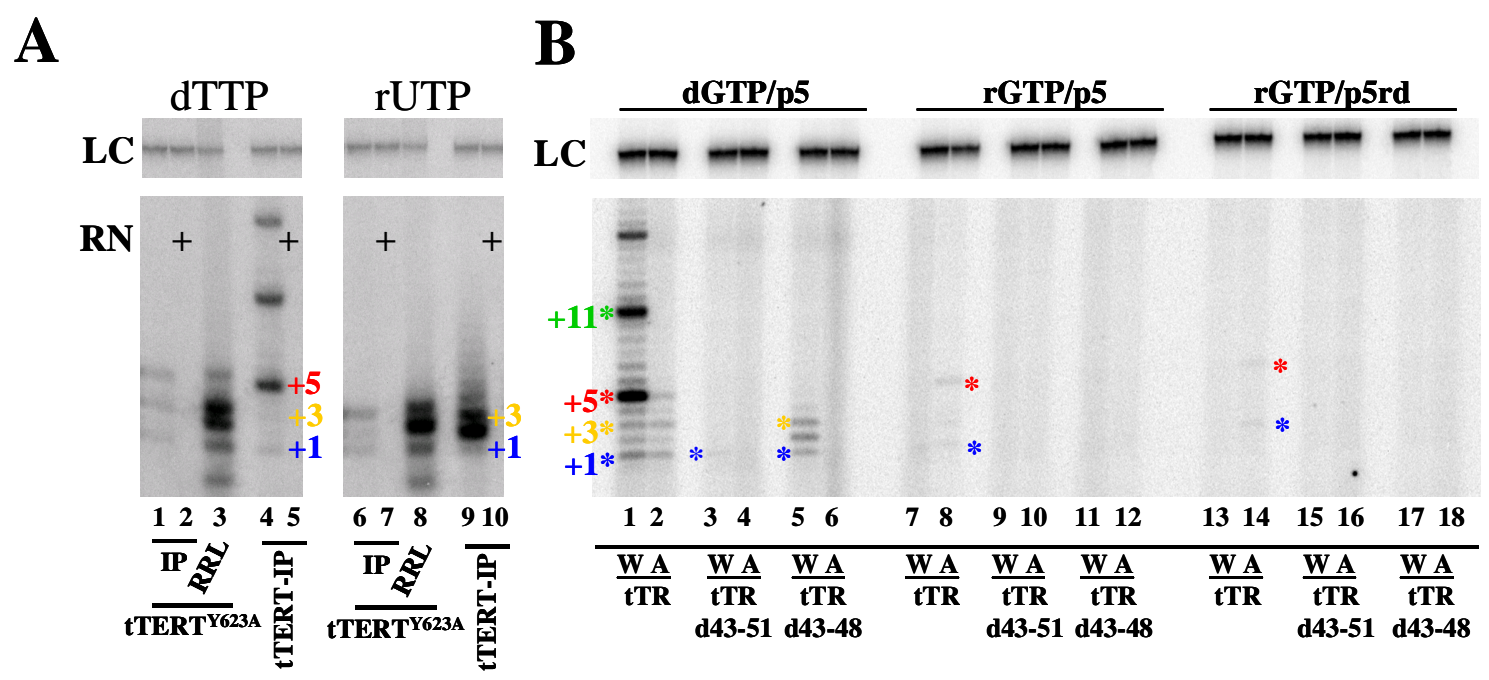

Figure S5: Telomerase-catalyzed extension of a chimeric primer using rGTP as the substrate. (A) Primer extension assay under standard conditions with p5 primer except $100 \mu \mathrm{M}$ rUTP (lanes 6-10) was substituted for dTTP (lanes 1-5) where indicated. Assays of immunoprecipitated tTERT ${ }^{\mathrm{Y} 623 \mathrm{~A}}$ (lanes 1, 2, 6, and 7) and tTERT (lanes 4, 5, 9, and 10), and crude tTERT ${ }^{\text {Y623A }}$ (lanes 3 and 8) each assembled with tTR are indicated. (B) Wild type tTERT (W; odd numbered lanes) or tTERT ${ }^{\mathrm{Y} 623 \mathrm{~A}}$ (A; even numbered lanes) were assembled with tTR (lanes 1, 2, 7, 8, 13, and 14), tTRd43-51 (lanes 3, 4, 9, 10, 15, and 16), or tTRd43-48 (lanes 5, 6, 11, 12, 17, and 18) then immunoprecipitated and assayed under standard reaction conditions with $10 \mu \mathrm{M}\left[\alpha-{ }^{32} \mathrm{P}\right]$-dGTP (Lanes 1-6) or [ $\alpha$ $\left.{ }^{32} \mathrm{P}\right]-$-rGTP (lanes 7-18), $2 \mu \mathrm{M}$ p5 (lanes 1-12) or p5rd (lanes 13-18), and $100 \mu \mathrm{M}$ dTTP (all lanes). Blue “*”, orange “*”, red “*”, and green “*” asterisks and/or numbers represent the extension of the indicated primer by one, three, five, and eleven nucleotides, respectively. $\mathrm{A}^{32} \mathrm{P}$-labeled loading control (LC) is indicated. Lanes labeled with $\mathrm{RN}$ are assays of samples pretreated with RNase. 